\title{
NN-SVG: Publication-Ready Neural Network Architecture Schematics
}

\section{Alexander LeNail ${ }^{1}$}

1 Massachusetts Institute of Technology, dept of Biological Engineering

DOI: $10.21105 /$ joss.00747

\section{Software}

- Review ¿

- Repository u

- Archive co

Submitted: 13 May 2018

Published: 15 January 2019

\section{License}

Authors of papers retain copyright and release the work under a Creative Commons Attribution 4.0 International License (CC-BY).

\section{Summary}

Illustrations of Neural Network architectures are often time-consuming to produce, and machine learning researchers all too often find themselves constructing these diagrams from scratch by hand.

NN-SVG is a tool for creating Neural Network (NN) architecture drawings parametrically rather than manually. It then provides the ability to export those drawings to Scalable Vector Graphics (SVG) files, suitable for inclusion in academic papers or as figures on web pages.

The tool provides the ability to generate figures of three kinds: classic Fully-Connected Neural Network (FCNN) figures, Convolutional Neural Network (CNN) figures of the sort introduced in (Lecun, Bottou, Bengio, \& Haffner, 1998), and Deep Neural Network figures following the style introduced in (Krizhevsky, Sutskever, \& Hinton, 2012). The former two are accomplished using the D3 javascript library (Bostock, Ogievetsky, \& Heer, 2011) and the latter with the javascript library Three.js. NN-SVG provides the ability to style the figure to the user's liking via many size, color, and layout parameters.

We hope this tool will save machine learning researchers time, and we hope this software might also serve as a pedagogical tool in some contexts.

\section{References}

Bostock, M., Ogievetsky, V., \& Heer, J. (2011). D3 data-driven documents. IEEE Transactions on Visualization and Computer Graphics, 17(12). doi:10.1109/TVCG.2011.185

Krizhevsky, A., Sutskever, I., \& Hinton, G. E. (2012). ImageNet classification with deep convolutional neural networks. In Advances in neural information processing systems 25. Retrieved from http://papers.nips.cc/paper/4824-imagenet-classification-with-deep-convolutionalpdf

Lecun, Y., Bottou, L., Bengio, Y., \& Haffner, P. (1998). Gradient-based learning applied to document recognition. In Proceedings of the ieee (pp. 2278-2324). 